\section{Kidney \\ Blood Pressure Research}

Kidney Blood Press Res 2015;40:277-287

DOI: $10.1159 / 000368503$

(C) 2015 S. Karger AG, Basel

www.karger.com/kbr

$1423-0143 / 15 / 0403-0277 \$ 39.50 / 0$

Accepted: March 03, 2015

This is an Open Access article licensed under the terms of the Creative Commons AttributionNonCommercial 3.0 Unported license (CC BY-NC) (www.karger.com/OA-license), applicable to the online version of the article only. Distribution permitted for non-commercial purposes only.

\title{
Diameter of Parathyroid Glands Measured by Computed Tomography as a Predictive Indicator for Response to Cinacalcet in Dialysis Patients with Secondary Hyperparathyroidism
}

\author{
Yu Ah Honga Yoo Sun Cho ${ }^{a}$ Sun Woo Kima Mi Yeon Junga Eun Ah Lee ${ }^{a}$ \\ Gang Jee Ko ${ }^{a}$ Heui Jung Pyo ${ }^{a}$ Soon Young Hwang ${ }^{b}$ Sangil Suh ${ }^{c}$ Young Joo Kwon ${ }^{a}$ \\ aDivision of Nephrology, Department of Internal Medicine; ${ }^{b}$ Department of Biostatistics; 'Department of \\ Radiology, Korea University College of Medicine, Seoul, Korea
}

\section{Key Words}

Cinacalcet • Parathyroid glands • Hyperparathyroidism, secondary • Computed Tomography - Ultrasonography

\begin{abstract}
Background/Aims: Cinacalcet is one of the important treatments of secondary hyperparathyroidism (SHPT). We evaluated the role of computed tomography (CT) for parathyroid glands (PTGs) to determine the response to cinacalcet therapy in dialysis patients. Methods: In study 1, we compared the predictive cutoff values of the largest volume or diameter of PTGs on ultrasonography or CT for achievement of target intact parathyroid hormone (iPTH) level according to K/DOQI guideline after cinacalcet treatment in a single dialysis center. In study 2, the role of the cutoff diameter of PTGs on CT in predicting responsive to cinacalcet therapy was reevaluated in dialysis patients with SHPT in multiple centers. Results: In study 1 , among the total population of 26 patients, the number of patients with baseline iPTH over 600 $\mathrm{pg} / \mathrm{mL}$ was 16 (61\%). In study 2, it was 45 (54\%), among 82 patients. In study 1, the number of PTGs equal to or larger than the cutoff value $(\geq 11.2 \mathrm{~mm})$ on CT, not ultrasonography, was significantly higher in non-responders than in responders $(p=0.038)$. In study 2 , the proportion of patients with PTGs $\geq 11.2 \mathrm{~mm}$ on CT was significantly higher in non-responders than responders $(p=0.003)$. Multivariate analysis showed that pretreatment iPTH (odds ratio [OR] 1.498, $p=0.003$ ) and the existence of enlarged PTGs on CT (OR 8.940, $p=0.015$ ) were significant clinical factors affecting the response to cinacalcet. Conclusions: The diameter of PTGs on CT could predict the response to cinacalcet in dialysis patients with SHPT.
\end{abstract}

Copyright $\odot 2015$ S. Karger AG, Basel 


\section{Kidney Blood Pressure Research}

Kidney Blood Press Res 2015;40:277-287

DOI: 10.1159/000368503

Published online: May 22, 2015

(C) 2015 S. Karger AG, Basel

www.karger.com/kbr

Hong/Cho/Kim/Jung/Lee/Ko/Pyo/Hwang/Suh/Kwon: Cinacalcet Response and Parathyroid Diameter on CT

\section{Introduction}

Secondary hyperparathyroidism (SHPT) is one of the most common complications in patients with chronic kidney disease (CKD) [1,2]. A variety of therapeutic or preventive strategies for SHPT are currently available, including dietary phosphate restriction, dialysis, calcium or non-calcium containing phosphate binders, non-selective and selective vitamin D receptor activators (VDRA), and calcimimetics.

Cinacalcet hydrochloride, an allosteric modulator of the calcium-sensing receptors (CaSRs), has played a role in the treatment of SHPT that is refractory to previous medical treatment. However, the response rate has been reported around 40-60\% [3-5], which means some patients of SHPT should seek another treatment such as parathyroidecomy bearing high cost for cinacalcet treatment and loss of time. In the point of view of cost-effectiveness, finding predictive indicator for response to cinacalcet therapy is clinically important. Several studies have been conducted to elucidate clinical factors related to the response to cinacalcet treatment in patients with SHPT, and the responsiveness to cinacalcet treatment depends on the number of enlarged parathyroid glands (PTGs) or PTG volume measured by ultrasonography (US) [6, 7], but these studies were done in relatively small population. Furthermore, Komaba et al. [8] suggested a significant reduction in PTG volume during treatment with cinacalcet in patients with SHPT regardless of pretreatment PTG volume on US. There is controversy as to whether PTG volume measured by US is capable of predicting the responsiveness to cinacalcet treatment in patients with SHPT.

Computed tomography (CT) is an objective tool for imaging studies that is fast, easily reproducible, and provides great sensitivity for accurate localization of hyperfunctioning PTGs. The relationship between PTG diameter measured by CT and response to cinacalcet treatment has not been established, and there are no comparisons of CT and US as reliable methods for PTG size measurement. We hypothesized that PTG diameter estimated by CT instead of US would be helpful in determining the response to cinacalcet treatment. The aim of this study was two-fold: (1) to elucidate whether measurement of PTG diameter by CT showed superiority over measurement of PTG volume or diameter by US as a predictor of response to cinacalcet therapy in a single center; and (2) to determine clinical and radiologic risk factors that influence the response to cinacalcet in patients with advanced SHPT in multi-centers.

\section{Patients and Methods}

\section{Study population and cinacalcet treatments}

This study adapted and re-analyzed the data of a study for the efficacy and safety of cinacalcet treatment in Korean hemodialysis (HD) and peritoneal dialysis (PD) patients with SHPT, which were published before (Kim et al.in HD patients and unpublished data in PD patients) [9]. It was designed as a prospective, open-label, single-arm study performed in Korea from September 2010 to December 2011. Patients over 18 years of age with stage 5 CKD who had received HD or PD for at least 3 months were enrolled. Eligible patients were required to have an albumin-corrected calcium level $\geq 9.0 \mathrm{mg} / \mathrm{dL}$ and an iPTH level $>300 \mathrm{pg} / \mathrm{mL}$ for two consecutive measurements before study initiation despite intensive treatment with VDRA and/or phosphate binders. The second iPTH measurement was performed at least 3 months after the first test, and there was no upper limit of serum iPTH level for enrollment. Subjects with a history of cinacalcet or bisphosphonate use, seizures, active infection, malignancy, or gastrointestinal bleeding, and those who were pregnant or breastfeeding at enrollment were excluded. The study was approved by the medical ethics committee of each hospital, and all participants provided written informed consent. All clinical investigations were conducted according to the principles expressed in the Declaration of Helsinki. The initial dose of cinacalcet was $25 \mathrm{mg} /$ day. We titrated the dose of cinacalcet in accordance with the serum iPTH, albumin-corrected calcium, and phosphorus levels measured every 2 weeks. The dose of cinacalcet was gradually increased to $100 \mathrm{mg}$ if serum iPTH was $>300 \mathrm{pg} / \mathrm{mL}$ and the albumin-corrected calcium level was $>8.5 \mathrm{mg} / \mathrm{dL}$. When the 


\section{Kidney Blood Pressure Research}

albumin-corrected calcium level was $<8.0 \mathrm{mg} /$ $\mathrm{dL}$ or the iPTH level was $\leq 150 \mathrm{pg} / \mathrm{ml}$, cinacalcet was titrated down or discontinued. After the initial 4- to 16-week titration period, the cinacalcet dose was maintained for 12 weeks to determine its efficacy. Serum calcium, phosphorus, and iPTH levels were measured every 4 weeks during the maintenance period. The type and dose of phosphate binders were flexibly adjusted according to the

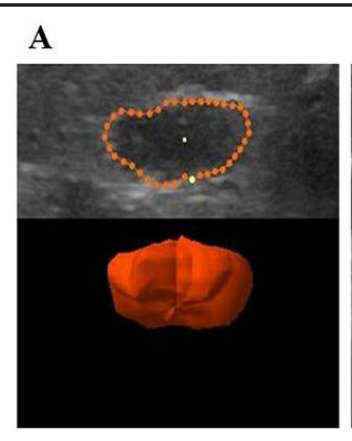

B

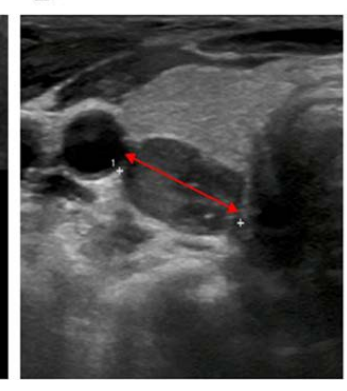

C

Fig. 1. Measurement of parathyroid gland (PTG) volume and diameter on high-resolution ultrasonography (US) and PTG diameter on computed tomography (CT) before cinacalcet therapy. A, B: Parathyroid gland volume and diameter measured by US. The PTG was diffusely hypoechoic and hypervascularized on color Doppler US. C: Measurement of parathyroid gland diameter by CT. physician's discretion.

\section{Study design and response of cinacalcet treatment}

This study was composed of two separate parts: In study 1, we determined the cutoff values of imaging parameters (PTG volume or largest diameter on US and CT) as the predictive indicator for cinacalcet response in a single center, In study 2, we reevaluated the clinical and radiologic risk factors for response of cinacalcet treatment in 13 university dialysis centers. The response of cinacalcet treatment was defined as a decrease in serum iPTH level $\leq 300 \mathrm{pg} / \mathrm{mL}$ during the study period. The response to cinacalcet was assessed for each patient during the treatment period, and patients were classified into two groups based on serum iPTH levels as follows: responders (group R), with iPTH level $\leq 300 \mathrm{pg} / \mathrm{mL}$ at least once during the study period, and non-responders (group NR), with iPTH level constantly $>300 \mathrm{pg} / \mathrm{mL}$.

\section{The clinical and biochemical parameters}

The clinical parameters were included as follows; age, sex, body mass index (BMI), diabetes mellitus, hypertension, dialysis modality, dialysis duration and blood pressure. Hemoglobin, serum albumin, calcium, phosphorus, alkaline phosphatase, iPTH levels, and lipid profile were measured at enrollment. Serum iPTH levels were measured using a radioimmunoassay kit (Cisbio Bioassays, Codolet, France, Normal ranges: 8-76 pg/mL). Serum calcium levels were corrected for albumin concentration using the following formula: [Corrected calcium=measured calcium $+(4-$ measured albumin $) \times 0.8]$.

\section{Parathyroid gland measurements}

To assess PTG size, high-resolution US (GE LOGIQ 6, General Electric Co., CT, USA) and CT (Aquilion ONE, Toshiba Medical Systems Corp., Tochigi, Japan) of the neck was performed before starting cinacalcet administration. Images of glands are shown in Figure 1. Ultrasonography was performed according to a standardized protocol using a 7.5- or 10-MHz linear array transducer. PTG volume was estimated using the ellipsoid formula $(\pi / 6 \times a \times b \times c$, where $a, b$, and $c$ represent the diameters of the gland in anterior-posterior, transverse, and longitudinal dimensions, respectively). PTG diameter and volume were measured by an experienced radiologist in a blinded manner; the largest value obtained was used in this study. In study 1 we evaluated PTG volume and diameter measured by US, and PTG diameter measured by CT. In study 2, PTG diameter was measured by CT.

\section{Statistical analysis}

All values are expressed as means \pm SD. We collected data for clinical and laboratory parameters and for largest PTG volume and diameters measured by US and CT. Differences between groups were analyzed by Mann-Whitney $U$ test for continuous nonparametric data, and by Fisher's exact test for categorical data. In study 1, Spearman`s correlation tests were used for correlation analyses. For calculation of receiver operating 


\section{Kidney \\ Blood Pressure Research}

Kidney Blood Press Res 2015;40:277-287

DOI: $10.1159 / 000368503$

Published online: May 22, 2015

(c) 2015 S. Karger AG, Basel

www.karger.com/kbr

Hong/Cho/Kim/Jung/Lee/Ko/Pyo/Hwang/Suh/Kwon: Cinacalcet Response and Parathyroid Diameter on CT

Table 1. Comparison of clinical and biochemical parameters between responders and non-responders to cinacalcet treatment in a single center (Study 1)

\begin{tabular}{lcccc}
\hline & $\begin{array}{c}\text { Total } \\
(\mathrm{n}=26)\end{array}$ & $\begin{array}{c}\text { Group R } \\
(\mathrm{n}=17)\end{array}$ & $\begin{array}{c}\text { Group NR } \\
(\mathrm{n}=9)\end{array}$ & p value \\
\hline Age (years) & $47 \pm 11$ & $44 \pm 10$ & $53 \pm 10$ & 0.055 \\
Gender, male (\%) & $7(26.9)$ & $4(23.5)$ & $3(33.3)$ & 0.661 \\
Dialysis duration (months) & $120 \pm 68$ & $126 \pm 79$ & $109 \pm 45$ & 0.936 \\
Hemoglobin (g/dL) & $10.3 \pm 1.7$ & $10.4 \pm 1.8$ & $9.9 \pm 1.5$ & 0.483 \\
Albumin (g/dL) & $3.9 \pm 0.5$ & $3.8 \pm 0.5$ & $4.0 \pm 0.5$ & 0.433 \\
Corrected calcium (mg/dL) & $10.4 \pm 0.8$ & $10.0 \pm 0.8$ & $10.8 \pm 0.8$ & $0.049^{*}$ \\
Phosphorus (mg/dL) & $5.8 \pm 1.2$ & $5.7 \pm 1.0$ & $6.0 \pm 1.5$ & 0.534 \\
ALP (IU/L) & $146.2 \pm 88.2$ & $117.6 \pm 35.9$ & $200.1 \pm 129.3$ & 0.138 \\
iPTH (pg/mL) & $837 \pm 415$ & $662 \pm 293$ & $1168 \pm 421$ & $0.002^{* *}$ \\
Largest volume of detectable PTG on US (mm $\left.{ }^{3}\right)$ & $821.9 \pm 942.0$ & $700.0 \pm 726.8$ & $1025.0 \pm 1245.9$ & 0.456 \\
Largest diameter of detectable PTG on US (mm) & $14.0 \pm 5.7$ & $14.0 \pm 5.6$ & $14.1 \pm 6.1$ & 0.952 \\
Largest diameter of detectable PTG on CT (mm) & $16.0 \pm 4.5$ & $15.5 \pm 4.2$ & $17.1 \pm 5.3$ & 0.138 \\
\hline
\end{tabular}

Differences between the values of group R and group NR analyzed by Mann-Whitney $U$ test and Fisher's exact test (gender). ${ }^{*} \mathrm{p}<0.05,{ }^{* *} \mathrm{p}<0.005$. R, responder; NR, non-responder; ALP, alkaline phosphatase; iPTH, intact parathyroid hormone; PTG, parathyroid gland; US, ultrasonography; CT, computed tomography; R, responder; $\mathrm{NR}$, non-responder, Responders (group R) were defined as iPTH level $\leq 300 \mathrm{pg} / \mathrm{mL}$ at least once during the study period, and non-responders (group NR) were defined as iPTH level constantly $>300 \mathrm{pg} / \mathrm{mL}$

characteristic (ROC) curves for the predictor variables of imaging parameters, the patients were stratified into responder (R) and non-responder (NR) groups. For each cutoff value curve, sensitivity was defined as the proportion of group $\mathrm{R}$ with predictor variables above the curve, and specificity as the proportion of group $\mathrm{NR}$ with values below the curve. The optimal cutoff value was defined as the cutoff obtaining the highest sum of sensitivity and specificity. In study 2, Wilcoxon signed rank test was performed for comparison between pre- and post-cinacalcet treatment data within R and NR groups. Logistic regression analysis was used to assess clinical factors that affected the therapeutic response to cinacalcet between group R and group NR. All analyses were performed using SPSS version 20 (IBM Corp., Armonk, NY, USA). A $P<0.05$ was considered statistically significant.

\section{Results}

\section{Study 1}

Comparison of imaging parameters as a predictive indicator for response to cinacalcet treatment in a single center. Study 1 included 26 dialysis patients who were treated with cinacalcet; 13 were undergone with HD and 13 with PD, and 17 were responders $(65.3 \%)$. Among the total population of 26 patients, the number of patients with iPTH over $600 \mathrm{pg} / \mathrm{mL}$ was $16(61 \%)$. Baseline characteristics are shown in Table 1. Age, gender, dialysis duration, hemoglobin, serum phosphate, and alkaline phosphatase values were not significantly different between group R and group NR. Serum iPTH and corrected calcium levels at baseline were lower in group R than in group NR (iPTH, $662 \pm 293 v s .1,168 \pm 421 \mathrm{pg} / \mathrm{mL}$, $\mathrm{p}=0.002$; corrected calcium, $10.0 \pm 0.8 v s .10 .8 \pm 0.8 \mathrm{mg} / \mathrm{dL}, \mathrm{p}=0.049$ ). During the screening period 24 of 26 patients underwent US and all 26 patients underwent CT. There was no significant difference in the mean volume and diameter of the largest PTG estimated by US and mean largest diameter measured by CT between group R and group NR at baseline. The difference in the largest diameter of PTG on US between group R and group NR was not significant. Similar to US, the largest diameter of PTG on CT is also not different between group R and group NR. The largest volume of PTGs on US was significantly correlated with the largest diameter of PTGs on US and CT in all patients by Spearman's correlation (PTG volume $v s$. PTG diameter on US; $r=0.767, \mathrm{p}<0.001$, PTG volume on US $v s$. PTG diameter on CT; $r=0.689, \mathrm{p}<0.001$ ). However, there was no significant correlation between PTG volume or diameter measured by US or CT and serum iPTH levels. 


\section{Kidney Blood Pressure Research}

Kidney Blood Press Res 2015;40:277-287

DOI: $10.1159 / 000368503$

Published online: May 22, 2015

(C) 2015 S. Karger AG, Basel

www.karger.com/kbr

Table 2. Comparisons of serum iPTH before and after cinacalcet treatment and change $(\Delta)$ in iPTH according to parathyroid gland length on CT or volume and length on US in a single center (Study 1)

\begin{tabular}{|c|c|c|c|}
\hline Gland & \multicolumn{2}{|c|}{ Number of PTGs } & $\mathrm{p}$ value \\
\hline \multirow{2}{*}{ 1) CT (length) } & \multirow{2}{*}{\multicolumn{3}{|c|}{$\begin{array}{cc}\text { larger than or equal to cutoff value }(11.2 \mathrm{~mm}) \\
0 & 1 \text { or more }\end{array}$}} \\
\hline & & & \\
\hline Pre-iPTH $(\mathrm{pg} / \mathrm{mL})$ & $465 \pm 55$ & $850 \pm 386$ & $<0.001^{* * *}$ \\
\hline Post-iPTH (pg/mL) & $187 \pm 37$ & $516 \pm 508$ & $0.010^{* *}$ \\
\hline$\Delta \mathrm{iPTH}(\mathrm{pg} / \mathrm{mL})$ & $-278 \pm 29$ & $-333 \pm 414$ & 0.560 \\
\hline \multirow{2}{*}{ 2) US (volume) } & \multirow{2}{*}{\multicolumn{3}{|c|}{$\begin{array}{cc}\text { larger than or equal to cutoff value }\left(475 \mathrm{~mm}^{3}\right) \\
0 & 1 \text { or more }\end{array}$}} \\
\hline & & & \\
\hline Pre-iPTH (pg/mL) & $764 \pm 363$ & $808 \pm 415$ & 0.786 \\
\hline Post-iPTH (pg/mL) & $361 \pm 402$ & $562 \pm 544$ & 0.315 \\
\hline$\Delta \mathrm{iPTH}(\mathrm{pg} / \mathrm{mL})$ & $-403 \pm 425$ & $-246 \pm 321$ & 0.318 \\
\hline \multirow{2}{*}{ 3) US (length) } & \multicolumn{3}{|c|}{ larger than or equal to cutoff value $(16.4 \mathrm{~mm})$} \\
\hline & 0 & 1 or more & \\
\hline Pre-iPTH (pg/mL) & $806 \pm 428$ & $745 \pm 290$ & 0.719 \\
\hline Post-iPTH (pg/mL) & $475 \pm 573$ & $435 \pm 218$ & 0.849 \\
\hline$\Delta \mathrm{iPTH}(\mathrm{pg} / \mathrm{mL})$ & $-331 \pm 408$ & $-311 \pm 331$ & 0.903 \\
\hline \multicolumn{4}{|c|}{$\begin{array}{l}\text { Values calculated by Mann-Whitney } U \text { test. }{ }^{*} \mathrm{p}<0.05,{ }^{* *} \mathrm{p}<0.005,{ }^{* * *} \mathrm{p}<0.001 . \mathrm{CT} \text {, } \\
\text { computed tomography; iPTH, intact parathyroid hormone; ultrasonography PTG, } \\
\text { parathyroid gland; US, }\end{array}$} \\
\hline
\end{tabular}

The cutoff values of PTG volume and diameter measured by US and PTG diameter measured by CT for response to cinacalcet treatment determined from the ROC curves were as follows: $475 \mathrm{~mm}^{3}$ for gland volume on US, $16.4 \mathrm{~mm}$ for PTG diameter on US, and $11.2 \mathrm{~mm}$ for PTG diameter on CT. Comparison of iPTH between groups divided by existence of PTGs equal to or larger than cutoff value of each modality, pre and posttreatment iPTH levels in the group with enlarged PTGs measured by CT were significantly higher than those in the group with no enlarged PTGs $(\mathrm{p}=0.01)$ (Table 2). However, there were no significant differences in pre and posttreatment iPTH values between the groups classified according to PTG volume or diameter measured by US. Table 3 shows that the proportion of patients with PTG diameter $\geq$ $11.2 \mathrm{~mm}$ measured by CT was significantly lower in group R than in group NR ( $p=0.038)$.

Study 2

Relationship between enlargement of parathyroid gland equal to or larger than the cutoff diameter on CT at baseline and response to cinacalcet treatment in multicenter study. Eighty-two out of 89 patients (56 HD patients and 33 PD patients) underwent CT during
Table 3. Number of enlarged parathyroid glands larger than or equal to cutoff value on CT scan according to response to cinacalcet in a single center (Study 1)

\begin{tabular}{cccc}
\hline $\begin{array}{c}\text { Number of enlarged } \\
\text { PTGs } \geq 11.2 \text { mm on CT }\end{array}$ & $\begin{array}{c}\text { Group } \\
\text { R }\end{array}$ & $\begin{array}{c}\text { Group } \\
\text { NR }\end{array}$ & p value \\
\hline 0 & $35.3 \%$ & $0 \%$ & $0.038^{*}$ \\
1 & $29.4 \%$ & $44.4 \%$ & \\
2 & $29.4 \%$ & $22.2 \%$ & \\
3 & $5.9 \%$ & $22.2 \%$ & \\
4 & $0 \%$ & $11.2 \%$ & \\
\hline
\end{tabular}

Values calculated by Fisher's exact test. ${ }^{*} \mathrm{p}<0.05$. CT, computed tomography; PTG, parathyroid gland; R, responder; NR, non-responder 


\section{Kidney \\ Blood Pressure Research}

Hong/Cho/Kim/Jung/Lee/Ko/Pyo/Hwang/Suh/Kwon: Cinacalcet Response and Parathyroid Diameter on CT

Table 4. Comparison of clinical characteristics and biochemical parameters between responders and non-responders to cinacalcet in a multicenter study (Study 2)

\begin{tabular}{lcccc}
\hline & $\begin{array}{c}\text { Total } \\
(\mathrm{n}=82)\end{array}$ & $\begin{array}{c}\text { Group R } \\
(\mathrm{n}=53)\end{array}$ & $\begin{array}{c}\text { Group NR } \\
(\mathrm{n}=29)\end{array}$ & $P$ value \\
\hline Age (years) & $48 \pm 12$ & $49 \pm 13$ & $45 \pm 10$ & 0.157 \\
Gender, male (\%) & $33(40.2)$ & $22(41.5)$ & $11(37.9)$ & 0.752 \\
BMI & $23.3 \pm 3.5$ & $23.1 \pm 3.6$ & $23.6 \pm 3.4$ & 0.585 \\
Diabetes (\%) & 15.9 & 19.2 & 10.3 & 0.296 \\
Hypertension (\%) & 84.1 & 82.7 & 89.7 & 0.398 \\
HD/PD & $53 / 29$ & $34 / 19$ & $19 / 10$ & 0.902 \\
Dialysis duration (months) & $108 \pm 68$ & $103 \pm 66$ & $116 \pm 72$ & 0.415 \\
Systolic BP (mmHg) & $137.9 \pm 22.5$ & $137.7 \pm 23.1$ & $138.2 \pm 21.9$ & 0.928 \\
Diastolic BP (mmHg) & $79.0 \pm 12.6$ & $77.7 \pm 12.7$ & $81.4 \pm 12.2$ & 0.201 \\
Hemoglobin (g/dL) & $10.9 \pm 1.5$ & $11.1 \pm 1.6$ & $10.4 \pm 1.4$ & 0.062 \\
Albumin (g/dL) & $3.9 \pm 4.1$ & $4.0 \pm 0.4$ & $3.9 \pm 0.4$ & 0.302 \\
Total cholesterol (mg/dL) & $153.5 \pm 36.0$ & $154.9 \pm 36.7$ & $150.9 \pm 35.2$ & 0.636 \\
LDL cholesterol (mg/dL) & $80.8 \pm 27.6$ & $80.1 \pm 28.2$ & $82.0 \pm 27.0$ & 0.802 \\
Triglyceride (mg/dL) & $118.6 \pm 71.6$ & $111.1 \pm 71.5$ & $132.4 \pm 71.2$ & 0.214 \\
Corrected calcium (mg/dL) & $10.1 \pm 0.9$ & $10.0 \pm 0.8$ & $10.3 \pm 1.0$ & 0.180 \\
Phosphorus (mg/dL) & $5.5 \pm 1.6$ & $5.4 \pm 1.6$ & $5.7 \pm 1.6$ & 0.355 \\
ALP (IU/L) & $245.1 \pm 358.8$ & $232.4 \pm 381.6$ & $267 \pm 319.1$ & 0.672 \\
iPTH (pg/mL) & $826 \pm 801$ & $631 \pm 311$ & $1214 \pm 1214$ & $0.023^{*}$ \\
\hline
\end{tabular}

Differences between the values of group R and group NR analyzed by Mann-Whitney $U$ test and Fisher's exact test (gender, diabetes, hypertension, and HD/PD). ${ }^{*} P<0.05,{ }^{* *} P<0.005,{ }^{* * *}$ $P<0.001$. ALP, alkaline phosphatase; BMI, body mass index; BP, blood pressure; iPTH, intact parathyroid hormone; LDL, low-density lipoprotein; R, responder; NR, non-responder; HD, hemodialysis; PD, peritoneal dialysis

the screening period and these were reanalyzed for study 2 . The clinical characteristics at baseline are shown in Table 4. Fifty-three patients were responders (64.6\%). The number of patients with iPTH over $600 \mathrm{pg} / \mathrm{mL}$ was $45 / 82$ (54\%). There were no significant differences in age, gender, BMI, prevalence of diabetes and hypertension, corrected calcium, phosphorus, alkaline phosphatase, and lipid profile between group R and group NR. Baseline serum iPTH was significantly lower in group R compared with group NR (iPTH; 631.1 \pm 311.4 vs. 1213.7 $\pm 1213.9 \mathrm{pg} / \mathrm{mL}, \mathrm{p}=0.023$ ).

Table 5 shows comparisons of biochemical parameters related to mineral and bone disorder between pre and posttreatment in each group. Cinacalcet treatment significantly decreased serum iPTH level in group $\mathrm{R}(\mathrm{p}<0.001)$. In contrast, although serum iPTH level also decreased after cinacalcet treatment in group NR, there was no statistical significance. Moreover, no significant changes were observed between pre and posttreatment phosphorus and alkaline phosphatase in group NR. Serum-corrected calcium was the only factor that significantly decreased after cinacalcet treatment in both group R and group NR.

To confirm whether our cutoff value for PTG diameter on CT was appropriate for predicting response of cinacalcet treatment, we divided the patients into two groups: patients with one or more enlarged PTG $\geq 11.2 \mathrm{~mm}$ and patients without enlarged PTG on CT. Figure 2A showed that serum iPTH level pre and posttreatment was significantly higher in patients with one or more enlarged PTG $\geq 11.2 \mathrm{~mm}$ than in patients without enlarged PTG on CT. The proportion of patients with PTG diameter $\geq 11.2 \mathrm{~mm}$ measured by CT was significantly higher in group NR than in group R by Fisher's exact test $(86.2 \%$ vs. $52.8 \%$, p $=0.003$, Figure 2B). We also investigated clinical factors that influence achievement of the target level of 


\section{Kidney Blood Pressure Research}
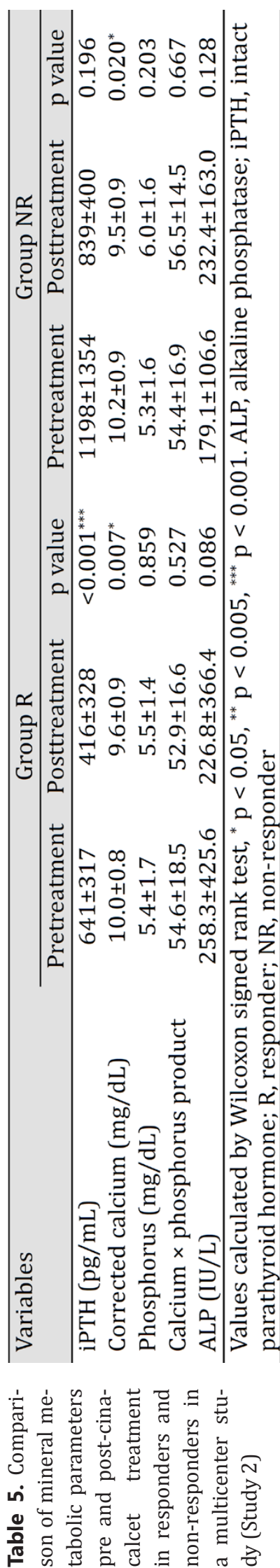

\section{Kidney Blood Press Res 2015;40:277-287}

DOI: 10.1159/000368503

Published online: May 22, 2015

C 2015 S. Karger AG, Basel

www.karger.com/kbr

Hong/Cho/Kim/Jung/Lee/Ko/Pyo/Hwang/Suh/Kwon: Cinacalcet Response and

Parathyroid Diameter on CT

Fig. 2. Comparison of iPTH level pre and post-cinacalcet treatment (A) and response rate to cinacalcet treatment (B) between patients with at least one enlarged PTG equal to or larger than the cutoff value measured by CT ( $\geq 11.2 \mathrm{~mm}$ ) and patients with no enlarged PTG (Study 2). $P$ values were calculated by Mann-Whitney $U$ test and Fisher's exact test, ${ }^{*}$ p value $<0.05,{ }^{* *} \mathrm{p}$ value $<0.005,{ }^{* * *} \mathrm{p}$ value $<0.001$, PTG, parathyroid gland; iPTH, intact parathyroid hormone.

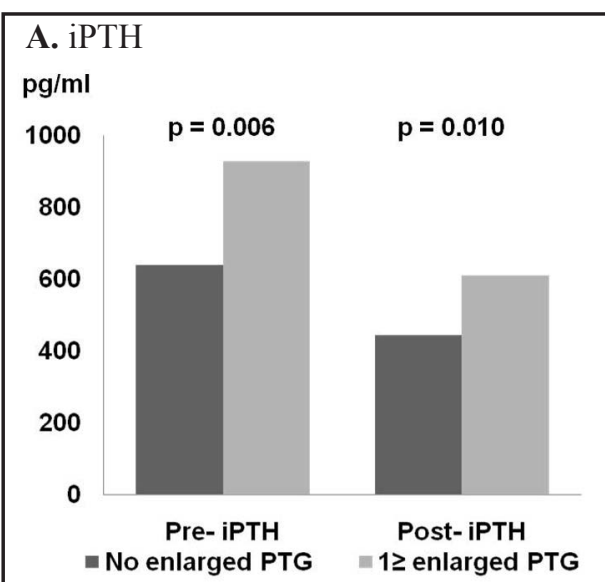

B. Response rate

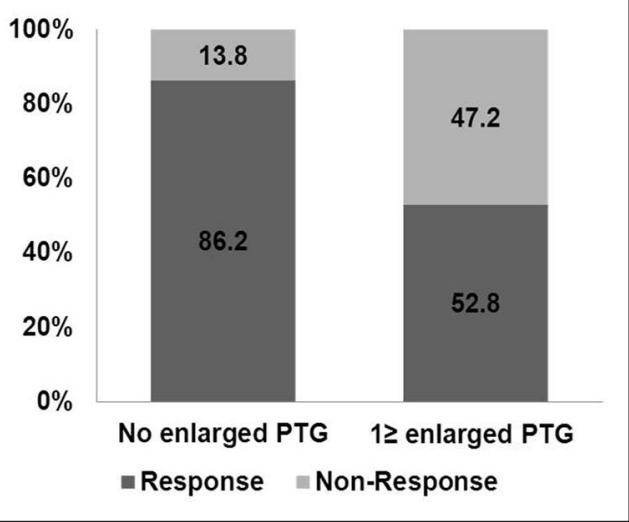

iPTH with cinacalcet treatment using a logistic regression model. In univariate logistic analysis, baseline iPTH and the existence of PTGs $\geq 11.2 \mathrm{~mm}$ on CT significantly influenced the response to cinacalcet treatment. In multivariate logistic analysis, the existence of enlarged PTGs equal to or larger than the cutoff value was the most significant factor for a poor cinacalcet response in SPHT (odds ratio [OR] 8.940, 95\% confidence interval [CI] 1.533-52.142, $\mathrm{p}=0.015$ ). Pretreatment iPTH level was also a significant clinical factor affecting achievement of response to cinacalcet treatment (OR 1.498, 95\% CI 1.15-1.952, p=0.003) (Table 6).

\section{Discussion}

We revealed that the existence of enlarged PTGs equal to or larger than the cutoff value on CT and a high iPTH level at baseline were significantly associated with a poor response to cinacalcet treatment. We proposed a specific cutoff value ( $\geq 11.2 \mathrm{~mm}$ ) from the ROC curve for PTG diameter on CT that predicts responsiveness to cinacalcet treatment.

The introduction of cinacalcet has led to a change in the treatment strategy for patients with SHPT who have 


\section{Kidney \\ Blood Pressure Research}

Table 6. Clinical factors influencing the response to cinacalcet treatment in SHPT in a multicenter study (Study 2)

\begin{tabular}{|c|c|c|c|c|}
\hline \multirow[t]{2}{*}{ Variable } & \multicolumn{2}{|l|}{ Univariate } & \multicolumn{2}{|c|}{ Multivariate } \\
\hline & Odds Ratio $(95 \% \mathrm{CI})$ & $\mathrm{p}$ value & Odds Ratio $(95 \% \mathrm{CI})$ & $\mathrm{p}$ value \\
\hline Age (per 1 year) & $0.972(0.935-1.011)$ & 0.158 & & \\
\hline Male & $1.161(0.459-2.937)$ & 0.752 & & \\
\hline Dialysis duration (per 1 year) & $1.003(0.996-1.010)$ & 0.411 & & \\
\hline Diabetes & $0.485(0.122-1.926)$ & 0.485 & & \\
\hline $\begin{array}{ll}\text { BMI } & \\
& <25 \\
& 25-30 \\
& >30\end{array}$ & $\begin{array}{c}0.400(0.026-6.176) \\
1.125(0.187-6.758) \\
1.600(0.231-11.082)\end{array}$ & 0.695 & & \\
\hline iPTH (per 100 pg/mL) & $1.304(1.111-1.530)$ & 0.001 & $1.498(1.15-1.952)$ & $0.003^{* *}$ \\
\hline Corrected Ca (per $1 \mathrm{mg} / \mathrm{dL}$ ) & $1.452(0.840-2.507)$ & 0.181 & & \\
\hline Phosphorus (per 1 mg/dL) & $1.146(0.861-1.526)$ & 0.351 & & \\
\hline ALP (per $1 \mathrm{IU} / \mathrm{L}$ ) & $1.000(0.999-1.002)$ & 0.670 & & \\
\hline $\begin{array}{l}\text { Existence of enlarged PTGs } \geq \\
11.2 \mathrm{~mm} \text { on CT }\end{array}$ & $5.58(1.706-18.257)$ & 0.004 & $8.940(1.533-52.142)$ & $0.015^{*}$ \\
\hline $\begin{array}{l}\text { Values calculated by logistic } r \\
\text { alkaline phosphatase; BMI, b } \\
\text { iPTH, intact parathyroid horm }\end{array}$ & $\begin{array}{l}\text { sion. }{ }^{*} \mathrm{p}<0.05, * * \mathrm{p} \\
\text { nass index; Ca, calci } \\
\text { PTG, parathyroid gla }\end{array}$ & $\begin{array}{l}05, * * \\
\text { T, col }\end{array}$ & $\begin{array}{l}\text { 0.001, CI, confidenc } \\
\text { ted tomography; HD, }\end{array}$ & $\begin{array}{l}\text { rrval, ALP } \\
\text { odialysis, }\end{array}$ \\
\hline
\end{tabular}

responded poorly to traditional treatment. Cinacalcet reduces the level of PTH without increasing calcium and phosphate, presumably as a consequence of reduced PTH-driven calcium and phosphate efflux from the bone [10]. Although cinacalcet has reduced PTH effectively in many cases, the efficacy of cinacalcet for achieving target iPTH in patients with SHPT according to K/DOQI guidelines was not sufficient, with a reported response rate to cinacalcet treatment of 43-62\% [3-5]. The precise mechanism underlying the unsatisfactory response to cinacalcet treatment is unclear. A recent retrospective study demonstrated that the extent of nodular hyperplasia is an important predictor of resistance to cinacalcet treatment in SHPT [11]. It is well known that PTGs with nodular hyperplasia are characterized by cells that are more closely packed together, which may lead to lower numbers of vitamin $D$ receptors and CaSRs, and a higher calcium set point for PTH secretion than in purely diffuse hyperplasia $[12,13]$.

Previous studies have suggested that enlarged PTGs are likely to represent nodular hyperplasia pathologically and that PTG size is also an important marker predicting the severity of SHPT $[14,15]$. Ultrasonography plays a pivotal role in diagnosis, assessment of disease severity, and evaluation of response to medical treatment for SHPT [16]. Nevertheless, US is usually operator-dependent, with high interoperator variability. Moreover, because PTG volume in SPHT is quite variable and there is no clear cutoff value for SHPT, the overall sensitivity of high-resolution US in the diagnosis of SHPT has been reported to be approximately 75\% [17]. In study 1, the number of PTGs equal to or larger than the cutoff value measured by CT was significantly related to the response to cinacalcet treatment. However, PTG diameter or volume measured by US did not show predictive value for response to cinacalcet treatment, even though the cutoff values of PTG volume and length on US determined in this study were similar to the previously proposed cutoff values of PTG volume $\left(>500 \mathrm{~mm}^{3}\right)$ and PTG diameter $(>10.0 \mathrm{~mm})$ for determining the response to cinacalcet treatment $[7,18]$. 


\section{Kidney Blood Pressure Research}

Kidney Blood Press Res 2015;40:277-287

DOI: $10.1159 / 000368503$

Published online: May 22, 2015

(C) 2015 S. Karger AG, Basel

www.karger.com/kbr

Hong/Cho/Kim/Jung/Lee/Ko/Pyo/Hwang/Suh/Kwon: Cinacalcet Response and Parathyroid Diameter on CT

To date, a few studies have been performed to elucidate clinical factors that influence the efficacy of cinacalcet on SHPT. A rapid decrease in iPTH levels after treatment and lower baseline serum iPTH levels were the main factors that were significantly associated with the response to cinacalcet treatment for SHPT $[9,19]$. In accordance with previous studies, we confirmed baseline serum iPTH as a strong predictive surrogate marker for response to cinacalcet treatment in patients with SHPT by multivariate logistic regression analysis. In addition, the presence of enlarged PTGs equal to or larger than the cutoff value measured by CT was significantly related to a poor response to cinacalcet treatment in this study. Computed tomography of the neck has low interoperator variability and has been used to localize enlarged PTGs with greater precision as a result of the improved resolution. Our research team recently reported that the maximum diameter of PTG detected on neck CT was highly correlated with the severity of SHPT based on iPTH [9]. This study showed that the largest diameter of PTGs measured by CT, rather than US, was sufficient to evaluate the efficacy of cinacalcet treatment in SPHT. Therefore, baseline diameter of PTGs measured by CT may help to predict cinacalcet response and guide to adapt another treatment for patients with SPHT that is refractory to cinacalcet therapy.

Previous randomized controlled studies have reported that cinacalcet-based treatment increased achievement of K/DOQI treatment targets in dialysis patients in whom conventional therapy was no longer effective for controlling SHPT [20, 21]. A recent subcohort study suggested that a combination of 'starting cinacalcet' and 'increased VDRA dose' was associated with better achievement of target iPTH levels than other treatments in cinacalcet-naïve patients [22]. Fukuma et al. [23] hypothesized that differences in the mechanisms of action between VDRA and cinacalcet may provide an additive clinical benefit in the management of SPHT. VDRA binds to vitamin D receptor and inhibits PTH synthesis in the PTG, whereas cinacalcet modulates calcium sensing receptors and inhibits PTH secretion. However, the combination treatment of 'starting cinacalcet' and 'increase VDRA dose' is extremely expensive costs for controlling SPHT. Based on the results of the present study, the existence of enlarged PTGs more than cutoff value in baseline imaging parameters indicates a need for early adaptation of alternative management strategies such as parathyroidectomy in anticipation of a poor response to cinacalcet treatment for avoiding waste of time and huge cost.

There are several limitations in this study. First, this study was performed with an openlabel design, and the duration of cinacalcet treatment was relatively short. Second, the sample size of the enrolled patients was relatively small. Therefore, a prospective, multicenter, largescale study with a longer follow-up period is required to more clearly define the relationship between the efficacy of cinacalcet treatment and imaging parameters. Last, we did not reevaluate PTG volume and diameters by CT or US to confirm the correlation between an improvement of iPTH and changes in imaging parameters after cinacalcet treatment.

\section{Conclusion}

We suggested that the diameter of PTGs measured by CT could predict the response to cinacalcet treatment in SHPT. The existence of enlarged PTGs equal to or larger than proposed cutoff values measured by CT and baseline iPTH levels could be useful predictors of therapeutic response to cinacalcet in SHPT.

\section{Disclosure Statement}

The authors have no financial and commercial conflicts of interest. 


\section{Kidney \\ Blood Pressure Research}

\section{Acknowledgements}

This study was supported in part by a grant from Kyowa Hakko Kirin Co., Ltd.. The company did not have any role in study design; collection, analysis, and interpretation of data; writing the report; or the decision to submit the report for publication. The authors would like to thank the following clinical Institution or Hospitals that participated in this study: Yonsei University College of Medicine (K. H. Choi); Keimyung University College of Medicine (H. C. Kim); The Catholic University of Korea College of Medicine (C. W. Park); Soonchunhyang University College of Medicine (S. D. Hwang); School of Medicine, Ewha Womans University (K. B. Choi); Inje University College of Medicine (Y. H. Kim); Chonnam National University Medical School (S. W. Kim); Kyung Hee University School of Medicine (T. W. Lee, J. Y. Moon); Pusan National University School of Medicine (I. S. Kwak); Aju University College of medicine (K. T. Shin); University of Ulsan College of medicine (J. S. Lee).

\section{References}

1 Martin KJ, Gonzalez EA: Metabolic bone disease in chronic kidney disease. J Am Soc Nephrol 2007;18:875885.

2 Rodriguez M, Nemeth E, Martin D: The calcium-sensing receptor: a key factor in the pathogenesis of secondary hyperparathyroidism. Am J Physiol Renal Physiol 2005;288:F253-264.

3 Block GA, Martin KJ, de Francisco AL, Turner SA, Avram MM, Suranyi MG, Hercz G, Cunningham J, AbuAlfa AK, Messa P, Coyne DW, Locatelli F, Cohen RM, Evenepoel P, Moe SM, Fournier A, Braun J, McCary LC, Zani VJ, Olson KA, Drueke TB, Goodman WG: Cinacalcet for secondary hyperparathyroidism in patients receiving hemodialysis. N Engl J Med 2004;350:1516-1525.

4 Block GA, Zeig S, Sugihara J, Chertow GM, Chi EM, Turner SA, Bushinsky DA: Combined therapy with cinacalcet and low doses of vitamin D sterols in patients with moderate to severe secondary hyperparathyroidism. Nephrol Dial Transplant 2008;23:2311-2318.

5 Moe SM, Chertow GM, Coburn JW, Quarles LD, Goodman WG, Block GA, Drueke TB, Cunningham J, Sherrard DJ, McCary LC, Olson KA, Turner SA, Martin KJ: Achieving NKF-K/DOQI bone metabolism and disease treatment goals with cinacalcet HCl. Kidney Int 2005;67:760-771.

6 Yamamoto M, Ogata H, Mizobuchi M, Yoshida N, Kumata-Maeta C, Koiwa F, Shishido K, Kinugasa E: Number of enlarged parathyroid glands might be a predictor of cinacalcet response in advanced secondary hyperparathyroidism. Clin Exp Nephrol 2012;16:292-299.

7 Hirai T, Nakashima A, Takasugi N, Yorioka N: Response of secondary hyperparathyroidism to cinacalcet depends on parathyroid size. Nephron Clin Pract 2010;114:c187-193.

8 Komaba H, Nakanishi S, Fujimori A, Tanaka M, Shin J, Shibuya K, Nishioka M, Hasegawa H, Kurosawa T, Fukagawa M: Cinacalcet effectively reduces parathyroid hormone secretion and gland volume regardless of pretreatment gland size in patients with secondary hyperparathyroidism. Clin J Am Soc Nephrol 2010;5:2305-2314.

9 Kim JK, Kwon YJ, Kim SW, Kim YH, Park CW, Choi KB, Hwang SD, Choi KH: Rapid decrease of intact parathyroid hormone could be a predictor of better response to cinacalcet in hemodialysis patients. Yonsei Med J 2013;54:453-463.

10 Verheyen N, Pilz S, Eller K, Kienreich K, Fahrleitner-Pammer A, Pieske B, Ritz E, Tomaschitz A: Cinacalcet hydrochloride for the treatment of hyperparathyroidism. Expert Opin Pharmacother 2013;14:793-806.

11 Hirai T, Nakashima A, Takasugi N, Yorioka N: Association of nodular hyperplasia with resistance to cinacalcet therapy for secondary hyperparathyroidism in hemodialysis patients. Ther Apher Dial 2010;14:577-582.

12 Fukuda N, Tanaka H, Tominaga Y, Fukagawa M, Kurokawa K, Seino Y: Decreased 1,25-dihydroxyvitamin D3 receptor density is associated with a more severe form of parathyroid hyperplasia in chronic uremic patients. J Clin Invest 1993;92:1436-1443. 


\section{Kidney \\ Blood Pressure Research}

Kidney Blood Press Res 2015;40:277-287

DOI: $10.1159 / 00036850$

(C) 2015 S. Karger AG, Basel

www.karger.com/kbr

Hong/Cho/Kim/Jung/Lee/Ko/Pyo/Hwang/Suh/Kwon: Cinacalcet Response and Parathyroid Diameter on CT

13 Gogusev J, Duchambon P, Hory B, Giovannini M, Goureau Y, Sarfati E, Drueke TB: Depressed expression of calcium receptor in parathyroid gland tissue of patients with hyperparathyroidism. Kidney Int 1997;51:328-336.

14 Fukagawa M, Kitaoka M, Yi H, Fukuda N, Matsumoto T, Ogata E, Kurokawa K: Serial evaluation of parathyroid size by ultrasonography is another useful marker for the long-term prognosis of calcitriol pulse therapy in chronic dialysis patients. Nephron 1994;68:221-228.

15 Okuno S, Ishimura E, Kitatani K, Chou H, Nagasue K, Maekawa K, Izumotani T, Yamakawa T, Imanishi Y, Shoji T, Inaba M, Nishizawa Y: Relationship between parathyroid gland size and responsiveness to maxacalcitol therapy in patients with secondary hyperparathyroidism. Nephrol Dial Transplant 2003;18:2613-2621.

16 Meola M, Petrucci I, Colombini E, Barsotti G: Use of ultrasound to assess the response to therapy for secondary hyperparathyroidism. Am J Kidney Dis 2011;58:485-491.

17 Meola M, Petrucci I, Calliada F, Barsotti M, Puccini M, Grosso M, Barsotti G: Presurgical setting of secondary hyperparathyroidism using high-resolution sonography and color Doppler. Ultraschall Med 2011;32:S7482.

18 Okada M, Tominaga Y, Izumi K, Nobata H, Yamamoto T, Hiramitsu T, Tsujita M, Goto N, Nanmoku K, Watarai T, Uchida K: Tertiary hyperparathyroidism resistant to cinacalcet treatment. Ther Apher Dial 2011;15:3337.

19 Segura Torres P, Borrego Utiel FJ, Sanchez Perales MC, Garcia Cortes MJ, Biechy Baldan MM, Perez Banasco $\mathrm{V}$ : [Analysis of the efficacy and factors influencing the response of secondary hyperparathyroidism patients on hemodialysis to cinacalcet]. Nefrologia 2010;30:443-451.

20 Fishbane S, Shapiro WB, Corry DB, Vicks SL, Roppolo M, Rappaport K, Ling X, Goodman WG, Turner $\mathrm{S}$, Charytan C: Cinacalcet $\mathrm{HCl}$ and concurrent low-dose vitamin D improves treatment of secondary hyperparathyroidism in dialysis patients compared with vitamin D alone: the ACHIEVE study results. Clin J Am Soc Nephrol 2008;3:1718-1725.

21 Messa P, Macario F, Yaqoob M, Bouman K, Braun J, von Albertini B, Brink H, Maduell F, Graf H, Frazao JM, Bos WJ, Torregrosa V, Saha H, Reichel H, Wilkie M, Zani VJ, Molemans B, Carter D, Locatelli F: The OPTIMA study: assessing a new cinacalcet (Sensipar/Mimpara) treatment algorithm for secondary hyperparathyroidism. Clin J Am Soc Nephrol 2008;3:36-45.

22 Fukagawa M, Fukuma S, Onishi Y, Yamaguchi T, Hasegawa T, Akizawa T, Kurokawa K, Fukuhara S: Prescription patterns and mineral metabolism abnormalities in the cinacalcet era: results from the MBD5D study. Clin J Am Soc Nephrol 2012;7:1473-1480.

23 Fukuma S, Kurita N, Fukagawa M, Akizawa T, Fukuhara S: Impact of cinacalcet introduction on MBD management: the MBD-5D study in Japan. Kidney Int Suppl (2011) 2013;3:436-441. 